\title{
Aerodynamic forces and flows of the full and partial clap-fling motions in insects
}

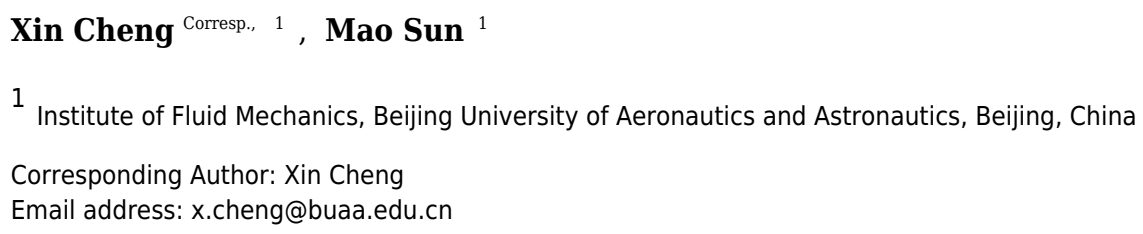

Most of the previous studies on Weis-Fogh clap-fling mechanism have focused on the vortex structures and velocity fields. Detailed pressure distribution results are provided for the first time in this study to reveal the differences between the full and the partial clapfling motions. The two motions are studied by numerically solving the Navier-Stokes equations in moving overset grids. The Reynolds number is set to 20 , relevant to the tiny flying insects. The following has been shown. (1) During the clap phase, the wings clap together and create a high pressure region in the closing gap between wings, greatly increasing the positive pressure on the lower surface of wing, while pressure on the upper surface is almost unchanged by the interaction; during the fling phase, the wings fling apart and create a low pressure region in the opening gap between wings, greatly increasing the suction pressure on the upper surface of wing, while pressure on the lower surface is almost unchanged by the interaction. (2) The interference effect between wings is most severe at the end of clap phase and the start of the fling phase: two sharp force peaks (8-9 times larger than that of the one-winged case) are generated. But the total force peaks are manifested mostly as drag and barely as lift of the wing, owing to the vertical orientation of the wing section. (3) The wing-wing interaction effect in the partial clap-fling case is much weaker than that in the full clap-fling case, avoiding the generation of huge drag. Compared with a single wing flapping with the same motion, mean lift in the partial case is enhanced by $12 \%$ without suffering any efficiency degradation, indicating that partial clap-fling is a more practical choice for tiny insects to employ. 
1 Aerodynamic forces and flows of the full and partial clap-fling motions in insects

4 Institute of Fluid Mechanics, Beijing University of Aeronautics \& Astronautics,

$5 \quad$ Beijing 100191, China

6

7 Corresponding Author:

$8 \quad$ Xin Cheng

$9 \quad$ NO.37, Xueyuan Road, Haidian District, Beijing, 100191, P.R.C. 


\section{Manuscript to be reviewed}

25

26

\section{Abstract}

Most of the previous studies on Weis-Fogh clap-fling mechanism have focused on the vortex structures and velocity fields. Detailed pressure distribution results are provided for the first time in this study to reveal the differences between the full and the partial clap-fling motions. The two motions are studied by numerically solving the Navier-Stokes equations in moving overset grids. The Reynolds number is set to 20, relevant to tiny flying insects. The following has been shown. (1) During the clap phase, the wings clap together and create a high pressure region in the closing gap between wings, greatly increasing the positive pressure on the lower surface of wing, while pressure on the upper surface is almost unchanged by the interaction; during the fling phase, the wings fling apart and create a low pressure region in the opening gap between wings, greatly increasing the suction pressure on the upper surface of wing, while pressure on the lower surface is almost unchanged by the interaction. (2) The interference effect between wings is most severe at the end of clap phase and the start of the fling phase: two sharp force peaks (8-9 times larger than that of the single-winged case) are generated. But the total force peaks are manifested mostly as drag and barely as lift of the wing, owing to the vertical orientation of the wing section. (3) The wing-wing interaction effect in the partial clap-fling case is much weaker than that in the full clap-fling case, avoiding the generation of huge drag. Compared with a single wing flapping with the same motion, mean lift in the partial case is enhanced by $12 \%$ without suffering any efficiency degradation, indicating that partial clap-fling is a more practical choice for tiny insects to employ. 


\section{Manuscript to be reviewed}

47

48

\section{Introduction}

Average insect wing length is about $3-4 \mathrm{~mm}^{1}$. Most of the previous studies, however, has focused on insects of relatively large size ${ }^{2-10}$. Tiny insects have a large population quantity and are of significant ecological and agricultural importance ${ }^{11-13}$, but their mechanics of flight is much less explored. Tiny insects fly at Reynolds number $(R e)$ below 60 where viscous effects are significant ${ }^{14-16}$. Previous numerical results of flapping wings have shown that the lift-to-drag ratio decreases greatly when $R e$ is below $\sim 100^{17-19}$. With the challenges associated with generating lift at such low Re, tiny insects must employ additional flight strategies to enhance lift, such as wing interference and wing flexibility. The most well-known example of beneficial interaction is the "clap and fling" mechanism proposed by Weis-Fogh ${ }^{14}$ for the small wasp Encarsia formosa in hovering. Towards the end of the upstroke, the wings clap together by rotating about their leading edges; then, at the beginning of the subsequent downstroke, the wings fling apart by rotating about their trailing edges.

Later observations of tiny insects' flight suggested that the clap and fling is a common phenomenon at this scale, for example, the greenhouse white-fly Trialeurodes vaoparium ${ }^{20}$, thrips ${ }^{15,21}$ and parasitoid wasp species Muscidifurax raptor and Nasonia vitripennis ${ }^{22}$. It is highly surprising that a marine mollusk, Limacina helicina also performs a "near fling" maneuver at stroke-reversal to augment lift $(R e=40-90)^{23}$. Larger insects rarely use clap and fling mechanism in 


\section{Manuscript to be reviewed}

69 free flight, with few exceptions, such as some moths in forward flight ${ }^{21}$, some butterflies in take-off flight ${ }^{24}$, and some locusts in climbing flight ${ }^{25}$. The small size and high wing beat frequency of tiny insects pose difficulty for video recording and the aforementioned tiny insects were all filmed with only one or two high-speed cameras. Consequently, no complete quantitative description of the clap and fling motion is available. Some progress in this field is made by Cheng and Sun ${ }^{16}$ who obtained detailed wing kinematics (including positional angle, deviation angle and pitch angle) of a small fly, vegetable leafminer Liriomyza sativae, hovering at $\operatorname{Re} \approx 40$. At dorsal stroke-reversal, the small fly has a partial clap-fling motion, which is a subtle variation of full clap-fling motion described for Encarsia formosa. More specifically, in the full clap-fling motion, both wings are parallel in close proximity along their entire surface at the dorsal stroke-reversal; in the partial clap-fling motion, only the outer parts of the wings are in close proximity and the wing roots are farther apart than the wing tips.

Inspired by Weis-Fogh's 1973 paper ${ }^{14}$, the lift-augmenting mechanism of the full clap-fling motion was widely studied by many researchers using theoretical methods ${ }^{26-30}$, physical models ${ }^{31-33}$ and numerical simulations ${ }^{15,22,34-41}$. In contrast, the partial clap-fling motion was thought to be just a variant of the full clap-fling motion and received much less attention ${ }^{16,42}$. All previous works supported the idea that the wing-wing interaction during clap-fling motion augments lift. For wings performing full clap-fling motion, the mean lift coefficient can be significantly enhanced by about $20 \%-70 \%{ }^{36,37}$, depended on different configurations of wing 
91 kinematic models; for wings performing partial clap-fling motion, the mean lift coefficient can be increased by about $7 \%-9 \%{ }^{16,42}$, indicating that partial clap-fling is somewhat less effective than the full clap-fling in enhancing lift.

However, the wing interference effects on drag force performances have been largely ignored in most previous studies. Based on the simulation results of the previous two- and three-dimensional computational works ${ }^{22,36,37}$, extremely large drag forces are produced in the full clap-fling cases at Reynolds numbers of tiny insects $(\operatorname{Re} \approx 10)$. For certain configurations, the drag required to fling the wings apart can be an order of magnitude larger than that required by a single wing with the same motion ${ }^{22}$. If so, the aerodynamic torque around the axis of azimuthal rotation, which is due to the drag force, will become too large for the wing hinge to support. Though can be reduced by about $50 \%$ with adding wing flexibility ${ }^{22}$ or increasing initial distance between wings ${ }^{37}$, the drag forces and relevant aerodynamic torques are still several times as large as those of the single wing. Using the measured wing motion data, Cheng and Sun ${ }^{16}$ calculated the aerodynamic forces of a tiny insect vegetable leafminer (it has a partial clap-fling motion at the dorsal stroke reversal). Their results showed that the drag forces with aerodynamic interaction are comparable to those without aerodynamic interaction, but they did not analyze the difference in detail. Lehmann et al. ${ }^{42}$ studied the partial clap-fling employing robotic fruit-fly wings $(\operatorname{Re} \approx 100)$ and showed similar results. Therefore, it is worthwhile to compare the aerodynamic performances between the full and the partial clap-fling motions in 3D circumstances and find out what causes the drag differences between 


\section{Manuscript to be reviewed}

113 the two cases.

The key feature of the partial clap-fling is that the wing separation varies along the wing span during clap-fling phase, it is predictable that the interference effect between wings decreases from wing tip to wing root. Given the radial dependency of flow down the span and the uniform application of wing separation in full clap-fling, it is interesting to figure out how the wing interference effect varies in the wing span direction with full clap and fling. Moreover, highlighting lift force only or discussing lift and drag force performances separately gives incomplete understanding of clap-fling mechanism. For the flapping wing in this study, the $R e$ is still high enough to assume that the aerodynamic force is almost normal to the wing surface and the lift and drag forces are components of the normal force perpendicular to and parallel to the stroke plane, respectively. It would be very helpful to firstly identify the underlying wing-wing interaction effect accounts for the total force difference, then the lift and drag force differences can be calculated depending on the orientation of the wing. clap-fling motion (constructed based on ref. 37) and the 3D partial clap-fling motion (constructed based on ref. 16). The aerodynamic forces and flow structure around model wings are computed by solving the Navier-Stokes equation numerically. The $R e$ is set to 20 . The interference effects are identified by comparing the results between single-winged and double-winged cases with the same wing motion. For the first time, we provide detailed pressure distribution results around wings during the 
135

136

137

138

139

140

141

142

143

144

145

146

147

148

149

150

151

152

153

complete clap-fling motion. How the "clapping together" and "flinging apart" of wings influence the fluids nearby is revealed by analyzing the flow fields and pressure distributions.

\section{Materials and Methods}

\section{Model wings and wing motions}

To compare the difference between the full and partial clap-fling motions, only one wing shape should be used in our simulation. As mentioned above, the full clap-fling motion constructed based on ref. 37 and the partial clap-fling motion constructed based on ref. 16 are used in the present study. We might either use the wing shape in ref. 37 or that in ref. 16. Here, we use the wing planform of the vegetable leafminer (VL1) in ref.16. The model wings and portions of computational grids are shown in figure 1. The wing section is assumed to be rigid flat plate. For small insects like fruitfly ${ }^{8}$ and vegetable leafminer ${ }^{16}$, their wings are small and relatively stiff so that the wing deformation is much smaller when compared with large insects like hawkmoth ${ }^{43}$. The aspect ratio $(A R)$, i.e. the ratio of the wing length $(R)$ to the mean chord length of the wing $(c)$, is 3.43. The radius of gyration $\left(r_{2}\right)$ of the model wing is $0.59 R$; the mean flapping velocity at the span location $r_{2}$ of the wing is defined as the reference velocity $U^{44}$ (defined below). 


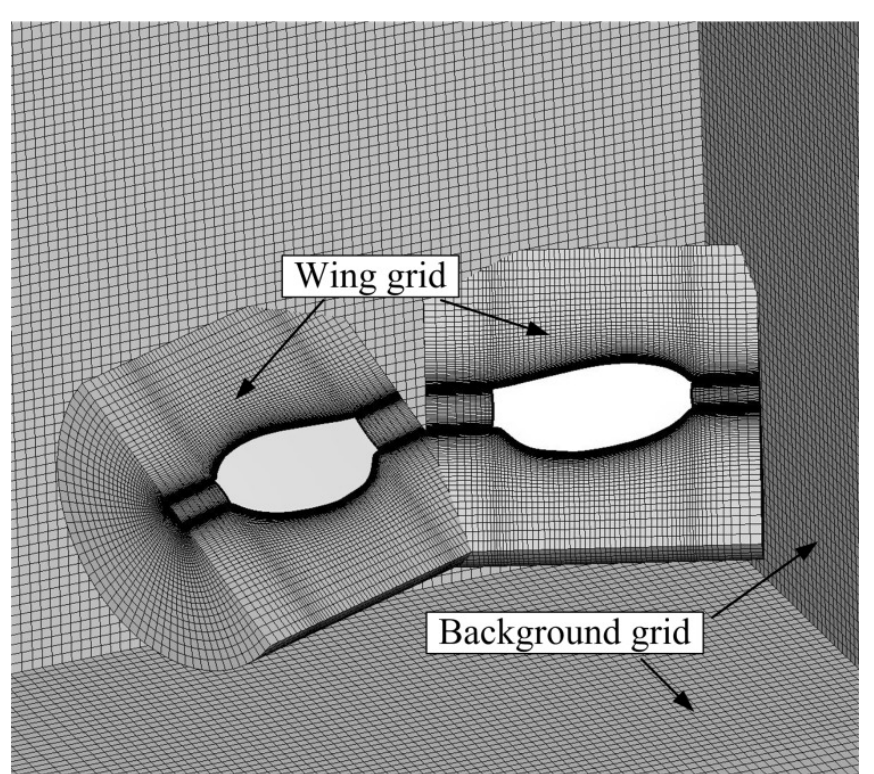

Figure 1. Model wings and portions of the computational overset grids.

Because the left wing is always the mirror image of the right wing during its motion, we only describe the motion of the right wing. To clearly describe the wing motion, two coordinate systems are introduced (Fig. 2a). Let $O X Y Z$ be an inertial frame with its origin at the wing root and oxyz be a non-inertial system with the same origin. For $O X Y Z, X$-axis and $Y$-axis form the horizontal plane (parallel to the stroke plane), and the $Z$-axis is vertical. oxyz is a frame fixed on the wing, with its $x$-axis parallel to the wing chord line and the $y$-axis along the wing span. Thus, the $z$-axis is normal to the wing surface. Based on the data given by Weis-Fogh ${ }^{14}$ and Ellington ${ }^{45}$, the full clap-fling motion is constructed as follows. The stroke cycle begins with the upstroke. Initially, the chord line of the wing is in vertical direction; at the start of the upstroke, the wing rotates around the flip axis ( $y$-axis); after the flip, the wing sweeps (rotates azimuthally around the Z-axis, referred as "translation") from the ventral to the dorsal side of the body; near the end of the upstroke, the wing rotates about its leading edge (the clap) and becomes vertical at the end of the upstroke; then the wing rotates about its trailing edge (the fling) at the beginning of the downstroke; following 
172 the fling, the wing sweeps from the dorsal to the ventral side of the body; near the end

173 of the downstroke, the wing rotates about the flip axis until its chord line is in vertical 174 direction again.

(a)
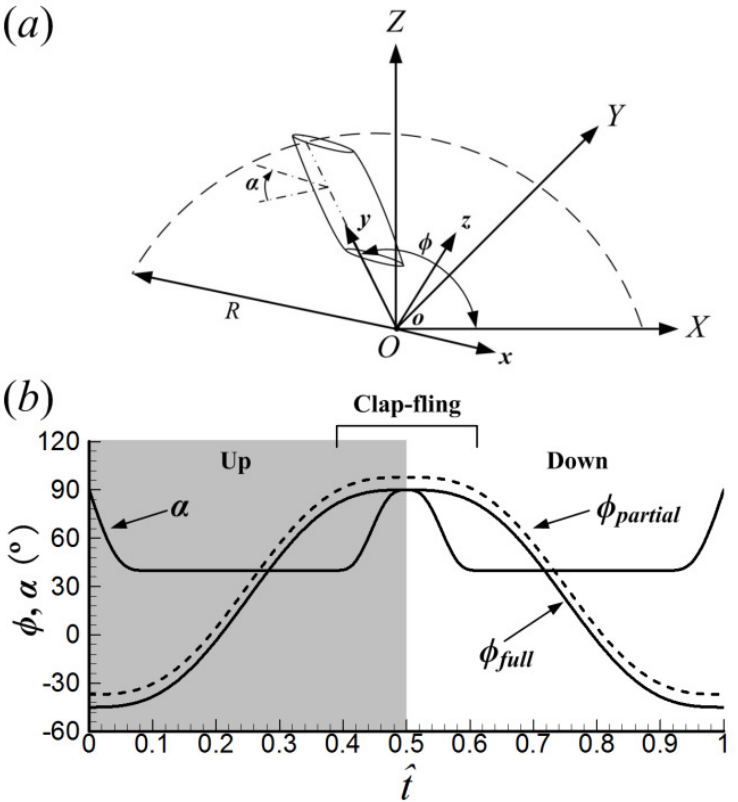

Figure 2. (a) Sketches of the reference frames and wing motion. (b) Time history of positional angle $(\phi)$ and angle of attack $(\alpha)$ in one cycle $\left(\phi_{f u l l}\right.$ represents the positional angle of the full clap-fling motion; $\phi_{\text {partial }}$ represents the positional angle of the partial clap-fling motion).

182 time variation of $\phi$ is approximated by a simple harmonic function:

184 where $\bar{\phi}$ is the mean stroke angle, $\Phi$ the stroke amplitude, $\tau$ the non-dimensional 185 time, and $\tau_{c}$ the non-dimensional period of the flapping cycle. $\bar{\phi}, \Phi, \tau$ and $\tau_{c}$ are defined as follows: $\bar{\phi}=\left(\phi_{\max }+\phi_{\min }\right) / 2, \Phi=\phi_{\max }-\phi_{\min }, \tau=t U / c$, and $\tau_{c}=U / c n$, where $\phi_{\max }$ is 
187 the maximum value of $\phi, \phi_{\min }$ the minimum value of $\phi, t$ the real time and $n$ the wingbeat frequency. The reference velocity $U$ is defined as $U=2 \Phi n r_{2}$. stroke plane (Fig. 2a), is assumed to be a constant value $\left(\alpha_{m}\right)$ in the mid-portion of a half-stroke except at stroke reversal (we call an up- or downstroke as a half-stroke). The ventral stroke reversal is a normal flip employed by most insects while the dorsal stroke reversal ("clap and fling") is a special flip with different $\alpha$ variation. The time variation of $\alpha$ during the flip rotation at the start of upstroke, the clap, the fling, and flip rotation at the end of downstroke are given as, respectively,

$\alpha=\alpha_{m}+0.5\left(\frac{\pi}{2}-\alpha_{m}\right)\left(1-\cos \left(\frac{\pi\left(\tau-0.5 \tau_{c}+\Delta \tau_{c l}\right)}{\Delta \tau_{c l}}\right)\right) \quad 0.5 \tau_{c}-\Delta \tau_{c l} \leq \tau \leq 0.5 \tau_{c}$

$\alpha=\frac{\pi}{2}-0.5\left(\frac{\pi}{2}-\alpha_{m}\right)\left(1-\cos \left(\frac{\pi\left(\tau-0.5 \tau_{c}\right)}{\Delta \tau_{f}}\right)\right) \quad 0.5 \tau_{c} \leq \tau \leq 0.5 \tau_{c}+\Delta \tau_{f}$

$\alpha=\alpha_{m}+\left(\frac{\pi}{2}-\alpha_{m}\right)\left(1-\cos \left(\frac{\pi\left(\tau-\tau_{c}+\Delta \tau_{r}\right)}{2 \Delta \tau_{r}}\right)\right) \quad \tau_{c}-\Delta \tau_{r} \leq \tau \leq \tau_{c}$

where $\Delta \tau_{r}$ is the flip rotation, $\Delta \tau_{c l}$ the clap duration and $\Delta \tau_{f}$ the fling duration. Here, $\tau_{r}$ is the time at which the flip rotation of the upstroke ends and $0.5 \tau_{c}$ is the time at which the upstroke ends.

The Reynolds number $(R e)$, which appears in the non-dimensional Navier-Stokes equations, is defined as $R e=c U / v$, where $v$ is the kinematic viscosity of the air. $R e$ is set to 20 in this paper. Based on the morphological data of the model wing used in the present study, the non-dimensional period is computed as follows: $\tau_{c}=U / c n=2 \Phi r_{2} / c=9.54$. The idealized full clap-fling motion used in this paper is 


\section{Manuscript to be reviewed}

208 similar to that used by Sun and $\mathrm{Yu}{ }^{37}$. We set $\phi_{\max }=90^{\circ}, \phi_{\min }=-45^{\circ}, \alpha_{\mathrm{m}}=40^{\circ}$,

$209 \Delta \tau_{r}=0.085 \tau_{c}, \Delta \tau_{c l}=\Delta \tau_{f}=0.11 \tau_{c}$. To clearly describe the wing motion, we express the

210 time during a cycle as a non-dimensional parameter $\hat{t}: \hat{t}=0$ at the start of an

211 upstroke and $\hat{t}=1$ at the end of the subsequent downstroke. The time courses of $\phi$ and

$212 \alpha$ of the right wing in one cycle is shown in Fig. 2b. For the full clap-fling motion, the

213 two wings are in the closest proximity at the end of upstroke (Fig. 3). Let $d$ denotes

214 the distance between wings at this instance (see Fig. 3, $\hat{t}=0.5$ ). In our previous study

$215^{16}$, the minimum distance between wing tips of VL1 was about $0.11 c$, very close to

$216 d=0.1 c$, which had been chosen in several previous numerical studies ${ }^{35-37,40}$. Therefore,

217 we specify $d=0.1 c$ in this study.

218 The partial clap-fling motion is constructed on the basis of the full clap-fling

219 motion described above by modifying two parameters: the distance between wing

220 roots and the positional angle $(\phi)$. More specifically, we obtain the partial clap-fling

221 motion by enlarging the distance between wing roots and introducing an angular

222 excursion of $+7.9^{\circ}$ to $\phi$ in the full clap-fling motion (the two values are decided based

223 on the wing kinematics of VL1 in our previous study ${ }^{16}$ ) (Fig. 2 b and Fig. 4). As a

224 result, in the partial clap-fling motion, only the outer parts of the wings are in close

225 proximity during clap and fling, the distance between wing tips at $\hat{t}=0.5$ is also

$226 d=0.1 c$. For comparison, a single wing performing identical motion as that of a wing

227 of the wing pair performing the full clap-fling motion is also considered. In this study,

228 only one flapping cycle is simulated.

229 

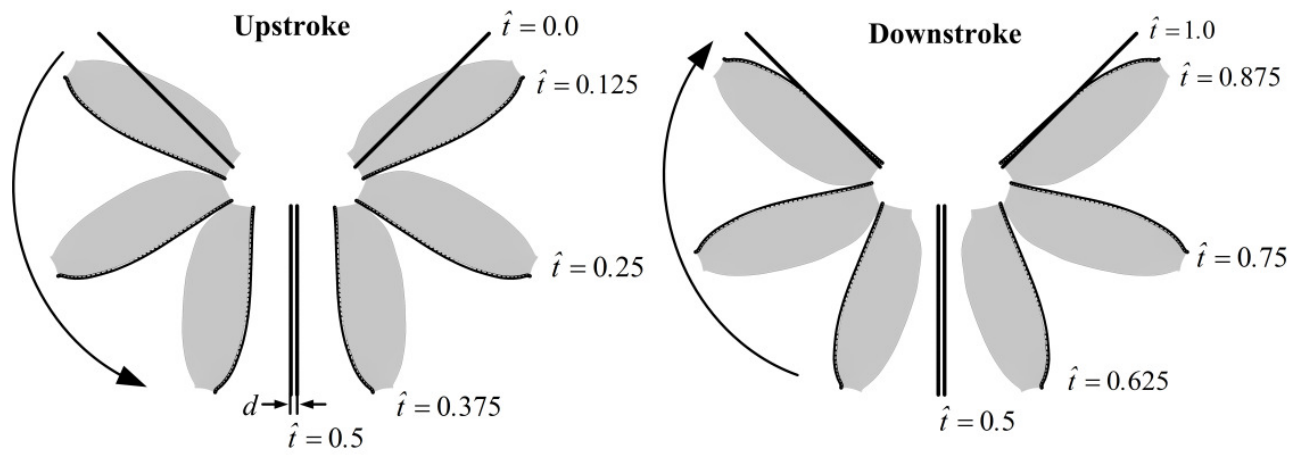

Figure 3. Schematic of the wing pair's motion (3D, top view) for the full clap-fling in a complete stroke cycle.

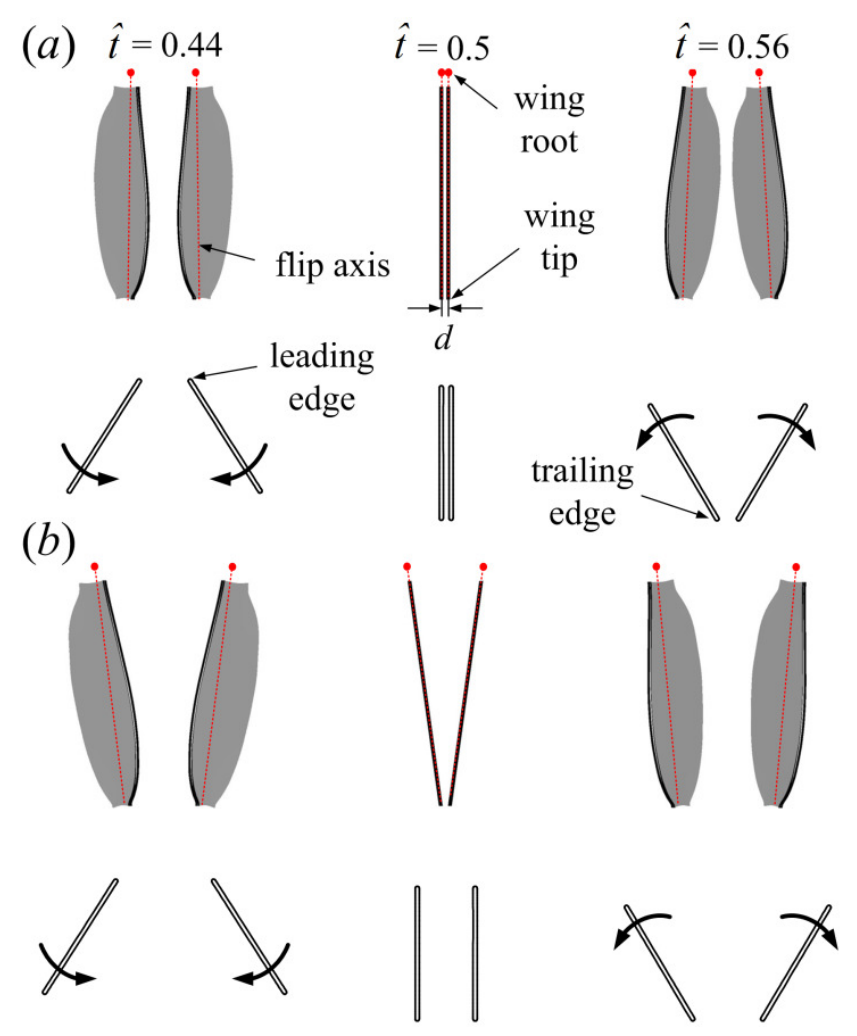

Figure 4. Motions of the wing pair (3D, top view) and the corresponding wing

237 clap-fling at three time instances $(\hat{t}=0.44, \hat{t}=0.5$ and $\hat{t}=0.56)$ during dorsal stroke 238 reversal. 


\section{Manuscript to be reviewed}

240

\section{Flow solution method and aerodynamic forces}

The flows around the wings are computed numerically by solving the Navier-

Stokes equations. In solving the Navier-Stokes equations, moving overset grids are used because the left and right wings are in close proximity during clap-fling motion, and there can be strong aerodynamic interactions between the wings. The numerical method is the same as that used by Sun et al. in several previous studies ${ }^{16,37}$. A description of it is given in the electronic supplementary material; the computational grids and grid resolution tests are also discussed there.

Solving the Navier-Stokes equations gives the fluid velocity components and pressure at discretized grid points for each time step. Here, the total aerodynamic force of a wing $(F)$ is computed by integrating the pressure and viscous stress over the wing surface. Lift $(L)$ is defined as the vertical component of the total aerodynamic force $(F)$ and $\operatorname{drag}(D)$ as the component that is in the horizontal plane ( $O X Y$ plane) (note that the $O X Y$ plane is coincide with the stroke plane). Normal force $(N)$ is the force normal to the wing surface and calculated by only integrating the pressure difference across the wing surface. For the flapping wing (thin wing operating at high angle of attack and the flow being separated), the normal force $(N)$ is almost identical to the total force $(F)$ and the lift $(L)$ and drag $(D)$ can be approximated by the components of the normal force $(N)$ normal to and parallel to the stroke plane, respectively. The corresponding coefficients of the above forces (denoted as $C_{F}, C_{N}, C_{L}$ and $C_{D}$, respectively) are defined as follows: $C_{F}=F / 0.5 \rho U^{2} S$, $C_{N}=N / 0.5 \rho U^{2} S, C_{L}=L / 0.5 \rho U^{2} S$ and $C_{D}=D / 0.5 \rho U^{2} S$, where $\rho$ is the fluid density and $S$ 
262 is the wing area.

\section{Results and discussion}

Using the wing motion data and numerical methods described in the Materials and Methods section, flows and aerodynamic forces of the full and the partial clap-fling cases were computed at $R e=20$, and the results were compared between the two cases and also with the corresponding single-winged case. For convenience, the full clap-fling case, the partial clap-fling case and the single-winged case are called FCF case, PCF case and SW case, respectively in the later discussions
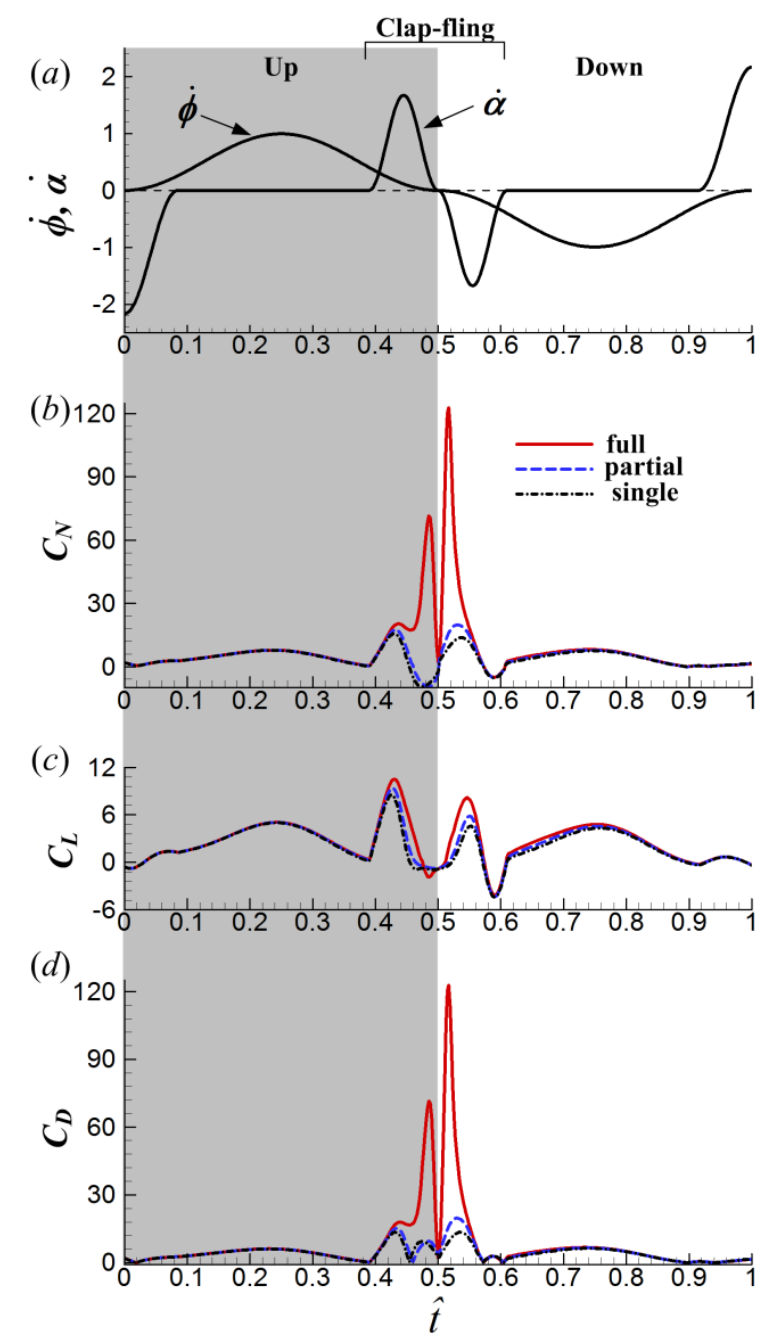


\section{Manuscript to be reviewed}

273 for the three different cases: (a) non-dimensional angular velocity of rotation ( \&)

274 and translation $(\$)$; (b) the normal force coefficient; (c) the lift coefficient; (d) the 275 drag force coefficient.

(1) Aerodynamic force differences

As aforementioned, normal force $(N)$ is the total force perpendicular to the wing

surface, lift $(L)$ can be approximated as the component of $N$ in the vertical direction

( $Z$ direction) and drag $(D)$ as the component in the stroke plane and normal to the

wing span. When the chord line of the wing is parallel to the stroke plane, i.e. $\alpha=0^{\circ}$,

the total normal force reorients in the lift direction; when the chord line is in the vertical direction, i.e. $\alpha=90^{\circ}$, the total normal force reorients in the drag direction.

Figure 5 gives the time courses of $C_{N}, C_{L}$ and $C_{D}$ in one cycle for the three different cases (the time histories of angular velocity $\$$ and \& are also given in the figure).

It is seen that, the interaction effect between wings is mostly restricted to the clap-fling phase $(\hat{t}=0.39-0.61)$ and decays rapidly once the wings move apart from each other. Moreover, $C_{N}$ of the FCF case is much larger than that of the PCF case in the mid-portion of the clap-fling phase $(\hat{t} \approx 0.45-0.55$, Fig. $5 a)$. And it should be noted that, the large $C_{N}$ obtained during the clap-fling phase in the FCF case is manifested mostly as $C_{D}$ rather than $C_{L}$ (compare the magnitude of $C_{L}$ and $C_{D}$ in Fig. $5 \mathrm{c}, \mathrm{d})$, especially at the end of the clap phase $(\hat{t}=0.48)$ and the start of the fling phase $(\hat{t}=0.52)$, the two sharp $C_{N}$ peaks are nearly equal to the two sharp $C_{D}$ peaks. 


\section{Manuscript to be reviewed}

295 reversal $(\hat{t} \approx 0.45-0.55)$, the pitch angle of attack $(\alpha)$ is large (see Fig. 2b) and the

296 wing plane is more vertical than that in the translation phase, so the total normal

297 force reorients more in the drag direction and less in the lift direction. In addition to

298 the sharp $C_{N}$ peak at $\hat{t}=0.48$ obtained only in the FCF case, there is another $C_{N}$ peak

299 at the start of clap phase $(\hat{t} \approx 0.43)$ which is obtained in all the three cases. This $C_{N}$

300 peak is produced by the "fast-pitching-up rotation" mechanism, proposed by Sun and

301 Tang ${ }^{46}$. In the early portion of clap $(\hat{t}=0.39-0.45$, see $\&$ in Fig. 5a) while

302 translating, the wing performs a fast pitching-up motion, generating strong vorticity

303 in a short time and hence large aerodynamic force ${ }^{8,46}$.

304 The two drag peaks of the FCF case are about 8-9 times as large as those in the

305 SW case at the same instances and cause a serious aerodynamic problem. To sweep

306 the wing back and forth in the stroke plane, the insect needs to overcome the

307 aerodynamic torque and inertial torque for translation (around the $Z$-axis) at the wing

308 root, while the aerodynamic torque is directly resulted from the drag force. In the

309 FCF case, the aerodynamic torques for translation at $\hat{t}=0.48$ and $\hat{t}=0.52$ also

310 become 8-9 times larger than those in the SW case, such large torque will be too

311 large for the wing hinge to support. In the PCF case, in contrast, the wing-wing

312 interaction effect is more moderate than that in the FCF case, avoiding the

313 generation of huge drag; there are only small quantitative differences in force

314 coefficients between the PCF case and the SW case (Fig. 5b-d). 


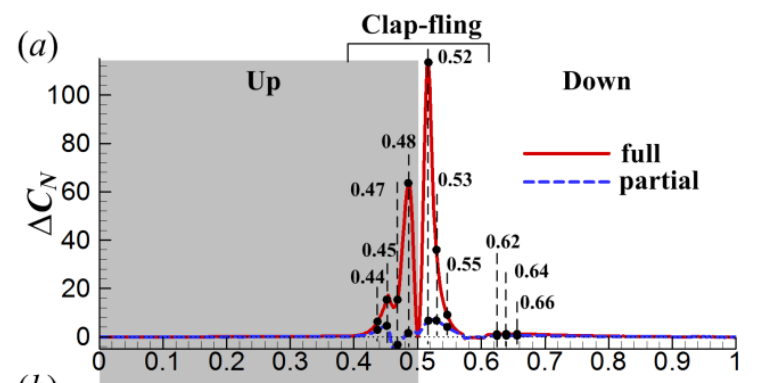

(b)
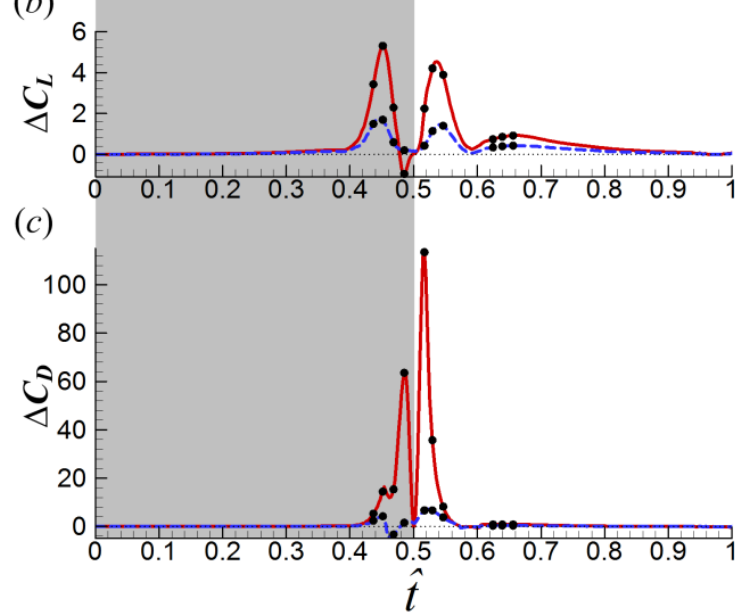

Figure 6. Time courses of difference in the normal force coefficient $\left(\Delta C_{N}\right)(\mathrm{a})$, lift coefficient $\left(\Delta C_{L}\right)(\mathrm{b})$ and drag coefficient $\left(\Delta C_{D}\right)$ (c) in one cycle between the one and two-winged cases (the ten small black dots indicate the instances at which detailed flowfield results were discussed).

To evaluate the influence of wing-wing interaction, the force differences between the one and two-winged cases were calculated (Fig. 6). As shown in Fig.6b, the interference effect makes the average $C_{L}$ during the clap-fling phase $(\hat{t}=0.39-0.61)$ 2.4 times and 1.5 times as large as that of the SW case in the FCF case and in the PCF case, respectively. Later in the downstroke after the fling phase $(\hat{t}=0.61-1.0)$, the lift-enhancing effect reduces rapidly but still slightly increases $C_{L}$ : average $C_{L}$ during this period increases $20 \%$ for the FCF case and $10 \%$ for the PCF case, compared with that of the SW case. Averaging $C_{L}$ and $C_{D}$ in one cycle $(\hat{t}=0.0-1.0)$ gives the 
329

330

331

mean lift coefficient $\left(\bar{C}_{L}\right)$ and mean drag coefficient $\left(\bar{C}_{D}\right)$ respectively. In the FCF case, $\bar{C}_{L}$ is increased by about $28 \%$ by the interaction, while $\bar{C}_{D}$ is approximately doubled compared to the SW case. Sun and $\mathrm{Yu}^{37}$ studied the 3D full clap-fling motion similar to that in the present work $(R e=15)$. Their computational results showed that $\bar{C}_{L}$ could be augmented by $20 \%$ and $\bar{C}_{D}$ be increased by $52 \%$, comparable to our results. In the PCF case, $\bar{C}_{L}$ is modestly enhanced, by about $12 \%$, and $\bar{C}_{D}$ is only increased by about $10 \%$. By employing robotic fruitfly wings $(R e \approx 100)$, Lehmann et al. ${ }^{42}$ studied the partial clap-fling motion similar to that in this paper. Their measurements showed that $\bar{C}_{L}$ could be augmented by about 9\%, similar to our results. If the aerodynamic efficiency is defined as the lift-to-drag ratio $\left(\bar{C}_{L} / \bar{C}_{D}\right)$, the full clap-fling motion appears to be rather inefficient. The partial clap-fling motion, however, can contribute more than $10 \%$ additional lift at tiny insect scale without suffering efficiency degradation. In this view, the partial clap-fling motion, rather than the full clap-fling motion, is a more practical choice for tiny insets to employ.

One interesting point to be noted in Fig. 6 is that, $C_{D}$ in the PCF case is even reduced somehow during the clap phase compared to the SW case $(\hat{t}=0.46-0.48$ in Fig. 6c), which would increase power efficiency of the PCF case. 


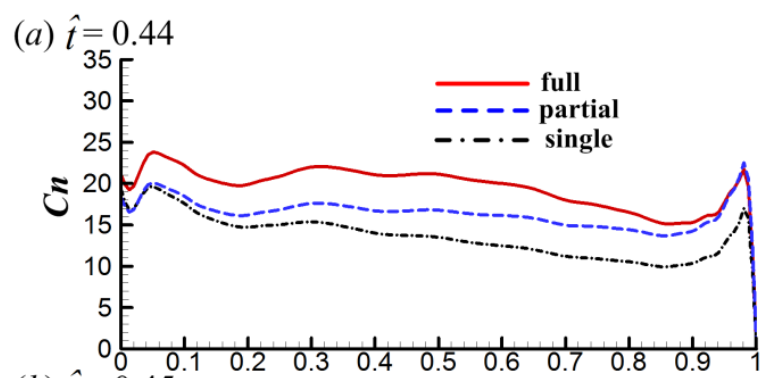

(b) $\hat{t}=0.45$

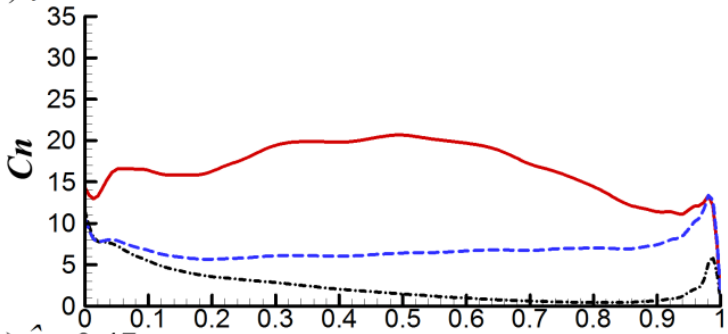

(c) $\hat{t}=0.47$

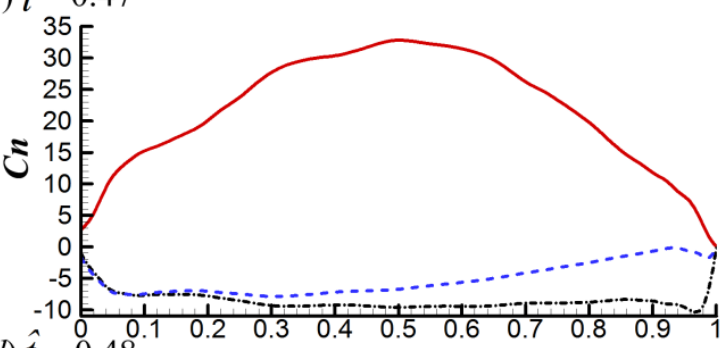

(d) $\hat{t}=0.48$

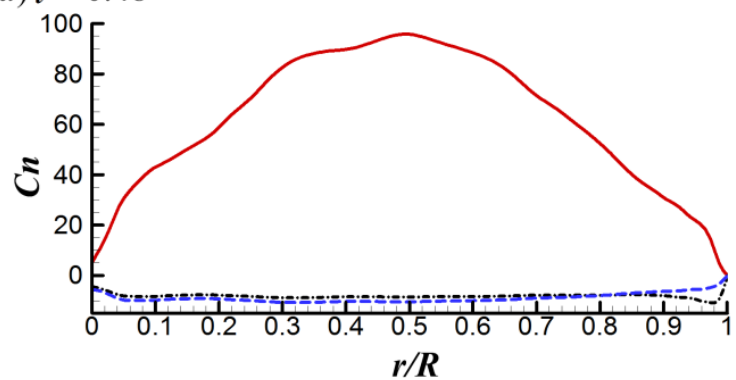

348 Figure 7. The spanwise distributions of the sectional normal force of the three

349 cases considered at $\hat{t}=0.44,0.45,0.47$ and 0.48 in the clap phase. 
(a) $\hat{t}=0.44$

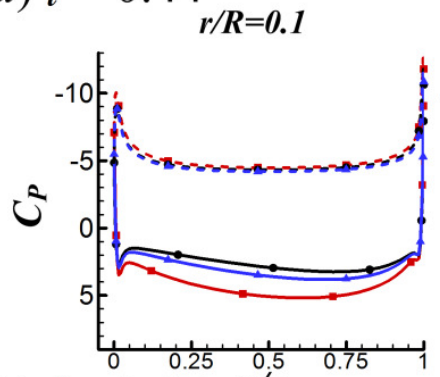

(b) $\hat{t}=0.45$
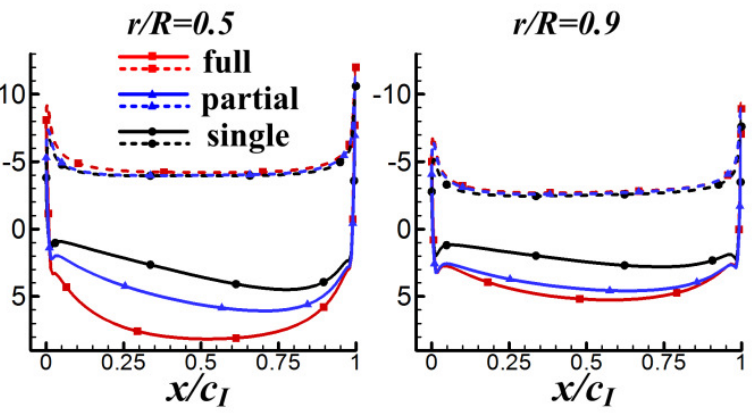

$r / R=0.1$

$r / R=0.5$

$r / R=0.9$

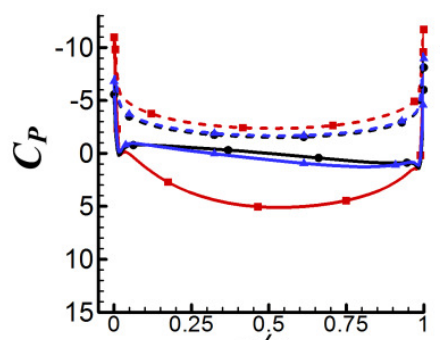

(c) $\hat{t}=0.47$
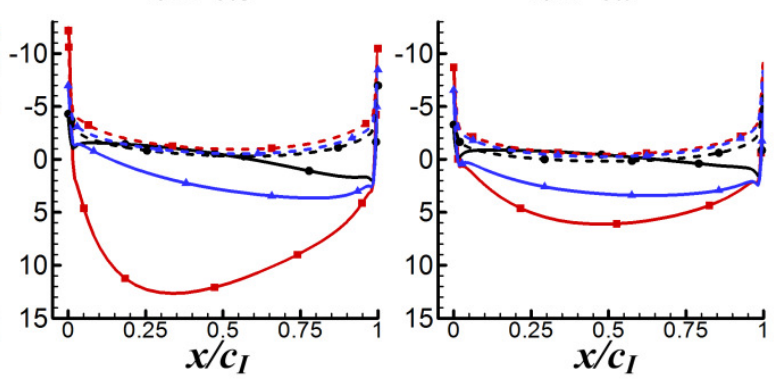

$r / R=0.1$

$r / R=0.5$

$r / R=0.9$

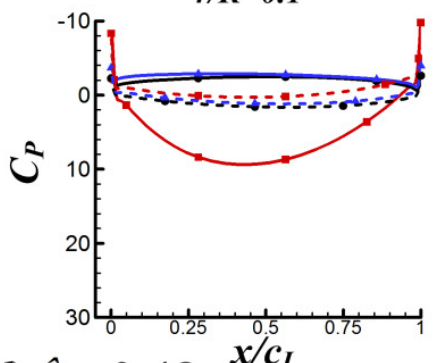

(d) $\hat{t}=0.48$
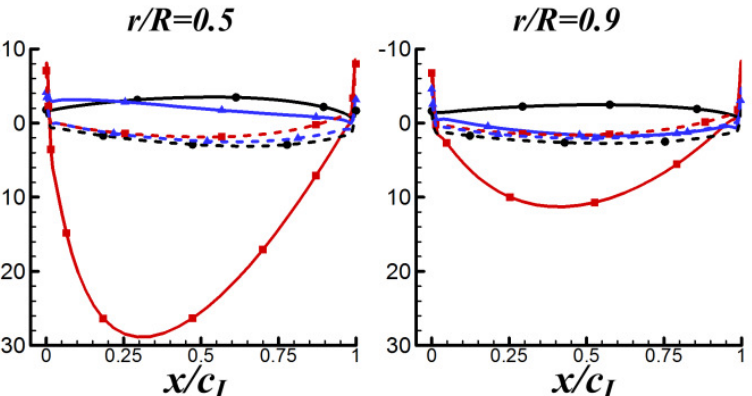

$r / R=0.1$

$r / R=0.5$

$r / R=0.9$
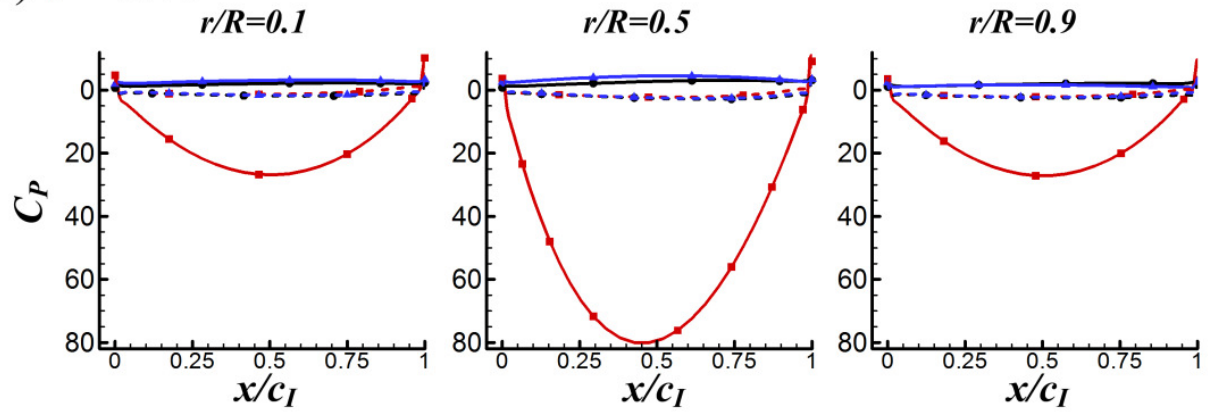

Figure 8. Surface pressure distributions of the three cases at various spanwise

352 positions for $\hat{t}=0.44,0.45,0.47$ and 0.48 in the clap phase (solid and broken lines

353 indicate the pressure distribution on the lower surface and the upper surface,

354 respectively). 
wing interaction in the FCF and PCF cases, the velocity fields and surface pressure

distributions at several instances are investigated (see Fig.6a, $\hat{t}=0.44,0.45,0.47$ and

0.48 in the clap phase; $\hat{t}=0.52,0.53$ and 0.55 in the fling phase and $\hat{t}=0.62,0.64$ and 0.66 in the subsequent translation after fling). How the underlying clap-fling mechanism influences the total force difference is discussed in the following.

First, we examine the clap phase $(\hat{t}=0.39-0.50)$. Figure 7 gives the corresponding sectional normal force distribution of the FCF, PCF and SW cases along the wing span at $\hat{t}=0.44,0.45,0.47$ and $0.48\left(C_{n}\right.$ denotes the coefficient of the sectional normal force and $r$ is the radial distance from the wing root). Figure 8 gives the surface pressure distribution of the three cases at several spanwise positions at the same time instants. $\left(C_{P}\right.$ denotes the pressure coefficient and is defined as $C_{P}=\left(p-p_{\infty}\right) / 0.5 \rho U^{2}$; solid and broken lines indicate the pressure distribution on the lower surface and the upper surface, respectively; $c_{I}$ denotes the local chord length of the wing at certain spanwise position). Note that the area enclosed by the curves representing the pressure coefficient $\left(C_{P}\right)$ in figure 8 is the non-dimensional sectional normal force $\left(C_{n}\right)$ at the specified spanwise position in

374 figure 7. During the clap phase $(\hat{t}=0.39-0.50)$, the positional angle $(\phi)$ is approximately constant (Fig. 2b) and the wing quickly rotates about the leading edge, respectively). 


\section{Manuscript to be reviewed}

379 case and the $C_{n}$ difference increases greatly with time. This is because, in the FCF

380 case, the two wings are in close proximity. The width of the gap between wings is

381 very small and does not change along the spanwise direction of wing, resulting in

382 strong interaction effect between wings. In contrast, the $C_{n}$ difference between the

383 PCF and the SW cases increases smoothly from wing root to wing tip: $C_{n}$ of the PCF

384 case is almost the same as that of the SW case at wing root and is almost the same as

385 that of the FCF case at wing tip. This is because, in the PCF case, the distance

386 between wing roots are much larger than that of wing tips. The wing roots are far

387 apart from each other so that the interaction effect is negligible, the distance between

388 wing tips is almost the same as that of the FCF case and hence the force-enhancing

389 effect. Comparing the $C_{P}$ distributions of the three cases in Fig. 8 shows that, $C_{P}$ is

390 almost the same on the upper wing surface (broken lines) while it varies significantly

391 on the lower wing surface (solid lines), indicating that the $C_{N}$ augmentation in the

392 two-winged cases is directly related to the higher lower-surface $C_{P}$ (with an

393 exception at $\hat{t}=0.47$, higher lower-surface $C_{P}$ of the PCF case results in smaller $C_{N}$

394 compared with the SW case, which is discussed later).

395 Next, how the higher lower-surface $C_{P}$ is produced by wing interaction is 396 explained and the difference between the FCF and PCF cases is compared. Figure 9 397 shows the velocity vectors and pressure distributions of the FCF case (a-d), the PCF 398 case (e-h) and the SW case (i-l) in vertical plane at half-wing length at the same four 399 instants as above. In the mid portion of clap $(\hat{t}=0.44$ and 0.45$)$, the wings move 400 towards each other quickly, the fluid between the wings is squeezed out of the 


\section{Manuscript to be reviewed}

401 closing gap and creates a high pressure region. Thus, the positive $C_{P}$ on the lower 402 surface of wing is greatly increased in the FCF case (Fig. 9a, b); because the wings 403 in the PCF case have larger separation distance, the $C_{P}$ enhancement on the lower 404 surface of wing becomes weaker (Fig. 9e, f). In the later portion of clap $(\hat{t}=0.47$ and 405 0.48), the whole wing surfaces in the FCF case are very close to each other, the high $406 C_{P}$ region in the very small gap between wings becomes much stronger than before 407 (Fig. 9c, d). In the PCF case, due both to the separation between wing roots and 408 rigidity of the model wing (Fig. 4b), only the outer parts of the wings near wing tip 409 are in close proximity, and the rest parts of the wings are still separated largely (Fig. $4109 \mathrm{~g}, \mathrm{~h})$. Meanwhile, the pitching angular-velocity around the leading edge of wing 411 decreases quickly to near zero at the end of clap phase (see \& in Fig. 5a). So the

412 interference effect in the PCF case is much weaker and nearly disappears at $\hat{t}=0.48$ 413 (Fig. 7d).

414 During $\hat{t} \approx 0.46-0.48$, due to the deceleration of pitching angular-velocity (see $415 \quad \&$ in Fig. 5a), $C_{N}$ of the PCF and SW cases becomes negative (see Fig. $5 \mathrm{~b}$ and Fig. 7c) and the absolute value of $C_{N}$ in the PCF case is even smaller than that of the SW 417 case, bringing benefit of $C_{D}$ reduction in the PCF case (see Fig. 6c). The reason for this is explained as following. The wing-wing interaction in the PCF case produces a

419 slightly higher lower-surface $C_{P}$ than that of SW case (see Fig. 8c). As a result, the 420 pressure difference between the lower and the upper surfaces becomes smaller and 421 hence the absolute value of $C_{N}$ (see Fig. 7c). Because $C_{N}$ obtained during this short period is manifested mostly as $C_{D}, C_{D}$ of the PCF case is reduced. 
423 Lower-surface pressure differences $\left(\Delta C_{P}\right)$ between the two two-winged cases

424 and the single-winged case at the specified four time instants are shown in figure 10,

425 which gives an overall picture of how the interaction effect affects $C_{P}$ on the whole

426 lower surface of wing. In the FCF case, $\Delta C_{P}$ is largest at the wing's center of area

427 and decreases form center to border (Fig. 10a-d); in the PCF case, the $C_{P}$

428 enhancement is much smaller compared to the FCF case and reaches its maximum

429 on the wing tip region (Fig. 10e-h).

430 
(a) $\hat{t}=0.44$
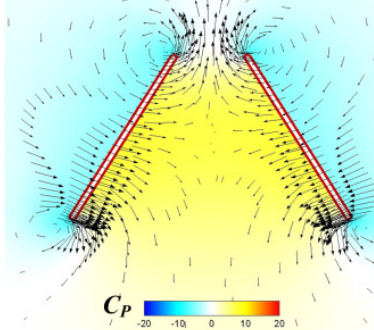

(b) $\hat{t}=0.45$

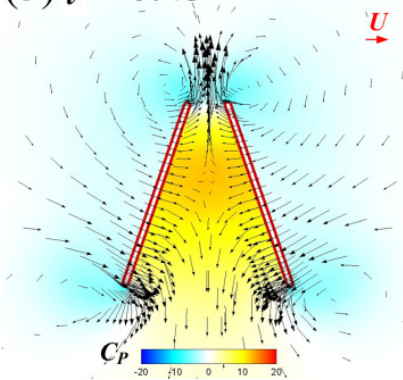

(c) $\hat{t}=0.47$

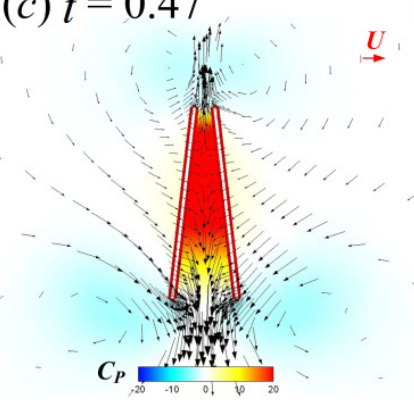

(d) $\hat{t}=0.48$

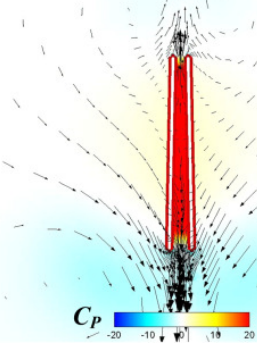

$\stackrel{U}{\rightarrow}$

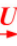

Partial

(e) $\hat{t}=0.44$

(i) $\hat{t}=0.44$

Single

$\stackrel{U}{\longrightarrow}$

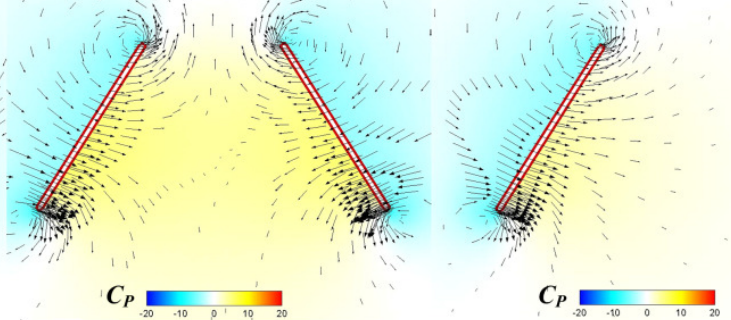

(j) $\hat{t}=0.45$

$\stackrel{U}{\rightarrow}$

(f) $t=0.45$

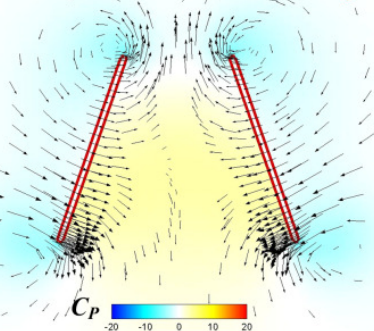

(g) $\hat{t}=0.47$

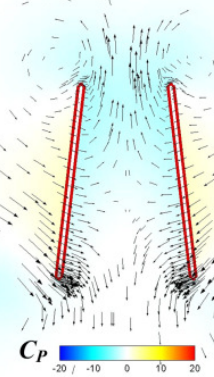

(k) $\hat{t}=0.47$

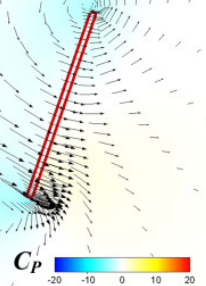

$\stackrel{U}{\rightarrow}$

${ }_{U^{\prime}}(h) \hat{t}=0.48$

(l) $\hat{t}=0.48$
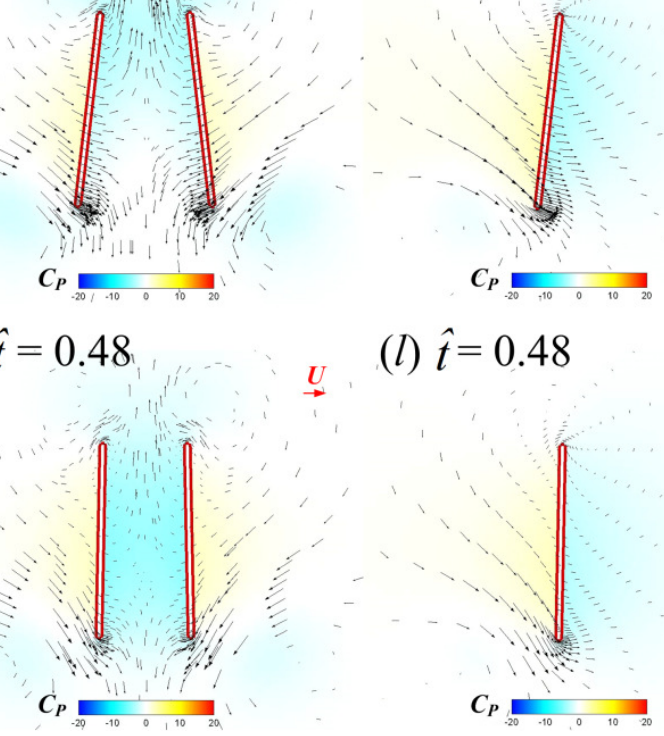

$\stackrel{U}{\longrightarrow}$

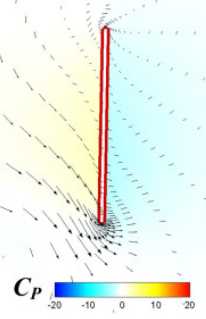

Figure 9. Velocity vectors and pressure distributions of the FCF case (a-d), PCF

433

case (e-h) and SW case (i-1) in vertical plane at half-wing length for $\hat{t}=0.44,0.45$,

434

0.47 and 0.48 in the clap phase (red horizontal arrow indicates reference velocity). 

Full
(a) $\hat{t}=0.44$
(e) $\hat{t}=0.44$
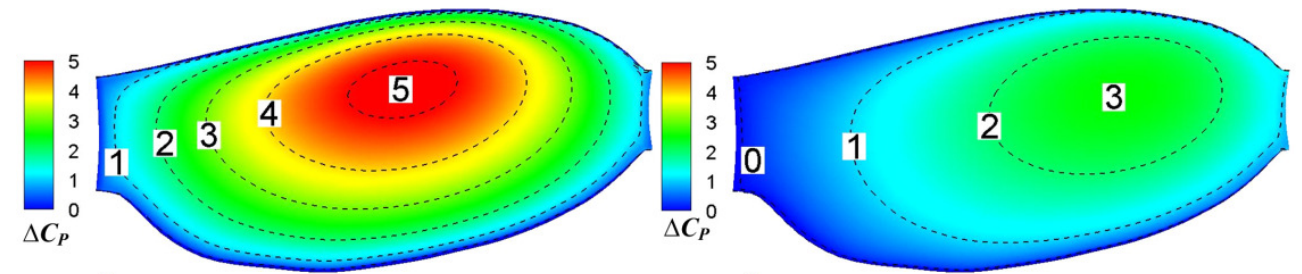

(b) $\hat{t}=0.45$

(f) $\hat{t}=0.45$
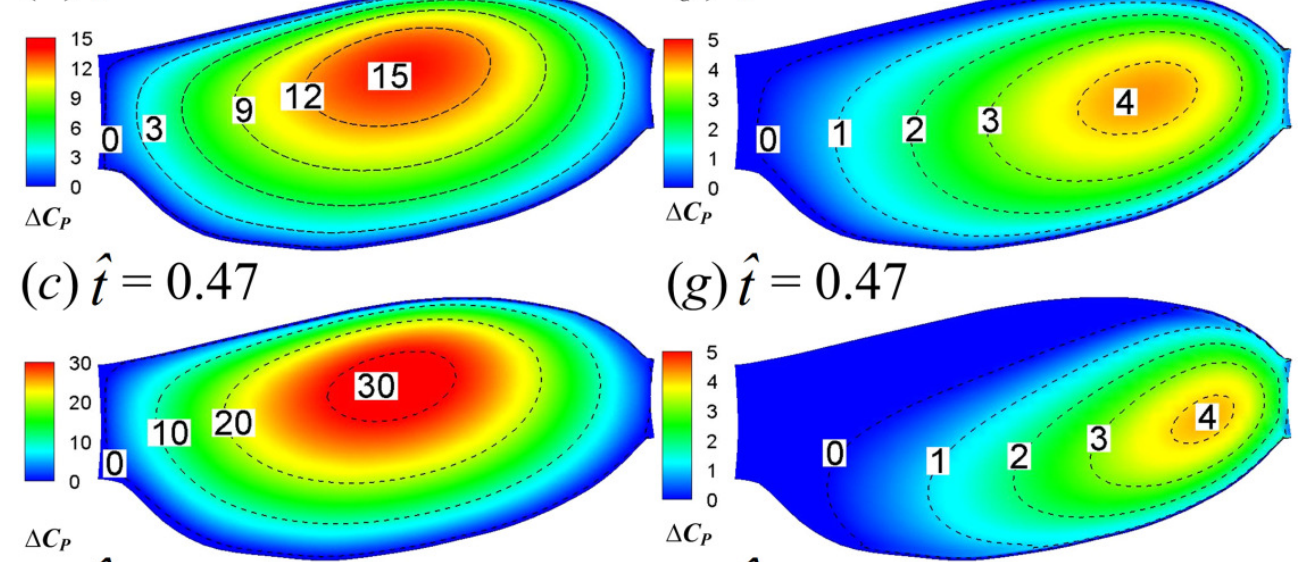

(g) $\hat{t}=0.47$

(d) $\hat{t}=0.48$

(h) $\hat{t}=0.48$
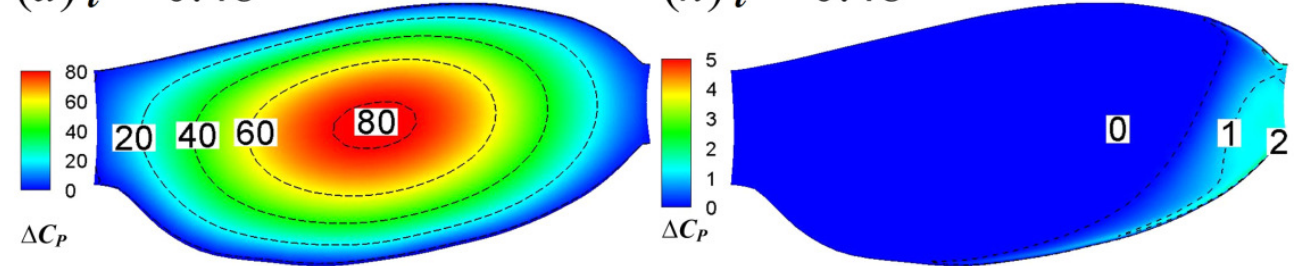

Figure 10. Lower-surface pressure differences between the two two-winged cases and the single-winged case at $\hat{t}=0.44,0.45,0.47$ and 0.48 in the clap phase. interaction, $\Delta C_{N}$ of the FCF case increases sharply to a peak value $(\hat{t}=0.52)$ immediately after the onset of fling and then decays rapidly in the later fling; in contrast, $\Delta C_{N}$ of the PCF case varies more gently (Fig. 6a). Figure 11 gives the spanwise distributions of $C_{n}$ at $\hat{t}=0.52,0.53$ and 0.55 of the three cases. We see that, in the FCF case, when the interaction effect is most obvious $(\hat{t}=0.52)$, The $C_{n}$ 


\section{Manuscript to be reviewed}

446 increment is largest at mid-span of wing and decreases gradually toward the sides

447 compared to the SW case (Fig. 11a); later in the downstroke, the $C_{n}$ increment does 448 not change greatly along the span (Fig. $11 \mathrm{~b}, \mathrm{c})$. In the PCF case, the $C_{n}$ increment is

449 smaller than that of FCF case all along the wing span, and it is relatively larger in the 450 outer part of the wing ( $r$ is large) than that in the inner part of wing ( $r$ is small). By 451 comparing the $C_{P}$ distributions in the three cases in Fig. 12, it is evident that the $C_{N}$ augmentation in the two-winged cases is attributed to the lower upper-surface $C_{P}$.

453 How the lower upper-surface $C_{P}$ is produced by wing interaction and the difference 454 between the FCF and PCF cases are discussed as follows. Figure 13 shows the 455 velocity vectors and pressure distributions of the FCF (a-c), PCF (d-f) and SW (g-i) 456 cases in vertical plane at half-wing length. During the fling motion, the wings fling 457 apart about the trailing edge. The gap between the wings is thus expanded and forms 458 a low pressure region, which accounts for the decrease of the upper-surface pressure. 459 At the onset of the fling motion ( $\hat{t}=0.52$ in Fig. 13), the gap between wings in the 460 FCF case is very small and a strong low pressure region is created in the gap when 461 the wing separation occurs (Fig. 13a); in contrast, the gap between wings in the PCF 462 case is large, which weakens the effect of wing-wing interaction, resulting in a weak 463 low pressure region in the gap (Fig. 13d). In the later portion of the fling $(\hat{t}=0.53$, 4640.55 in Fig. 13), the low pressure region draws fluid into the opening gap between 465 wings and forms a strong vortex near the leading edge. The leading edge vortex 466 (LEV) creates a low-pressure region and further reduces the amplitude of the 467 negative $C_{P}$ at leading edge, which explains why the largest negative $C_{P}$ is obtained 
468 at the leading edge at $\hat{t}=0.53$ and 0.55 (Fig. 12b, c). Moreover, an overall picture of

469 how the interaction effect varies on the whole upper surface of wing is shown in

figure 14, which illustrates the upper-surface pressure differences $\left(\Delta C_{P}\right)$ between the

471 two two-winged cases and the single-winged case at several distances during the

472 fling phase. In the FCF case, at the initial start of fling $(\hat{t}=0.52)$, the largest $C_{P}$

473 decrease is obtained at the wing's center of area (Fig. 14a); once the strong LEV is

474 formed, the largest $C_{P}$ decrease is obtained at the wing's leading edge (Fig. 14b, c).

475 In the PCF case, the amplitude of $C_{P}$ decrease is smaller and the position of largest $=0.53$ and 0.55 , but closer to the wing tip (Fig. $14 \mathrm{~d}-\mathrm{f}$ ).

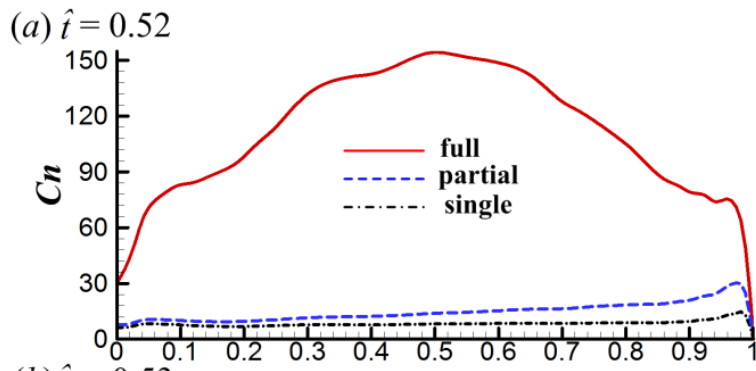

(b) $\hat{t}=0.53$

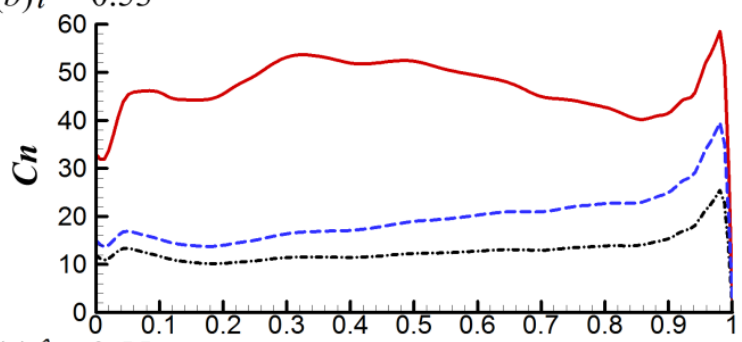

(c) $\hat{t}=0.55$

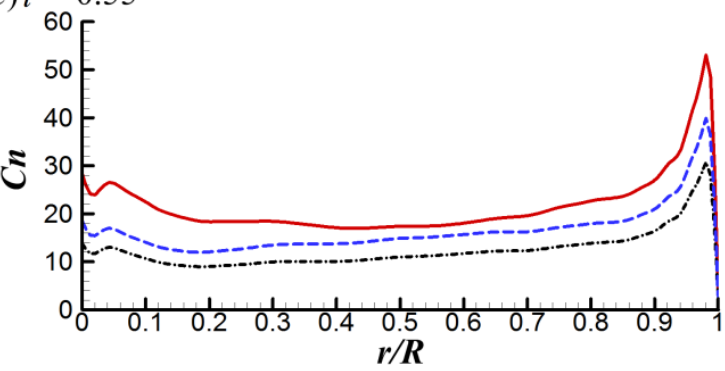

Figure 11. The spanwise distributions of the sectional normal force of the three cases considered at $\hat{t}=0.52,0.53$ and 0.55 . 
(a) $\hat{t}=0.52$

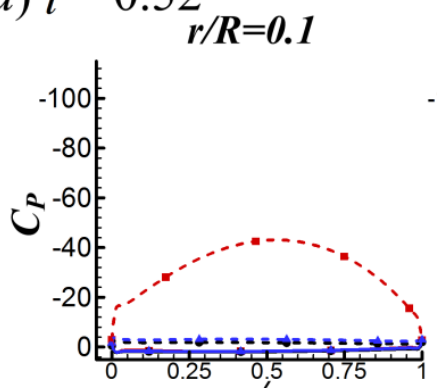

(b) $\hat{t}=0.53$
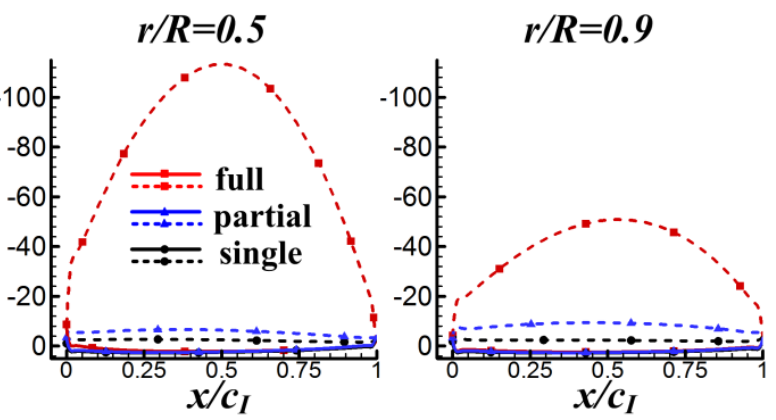

$r / R=0.5$

$r / R=0.9$
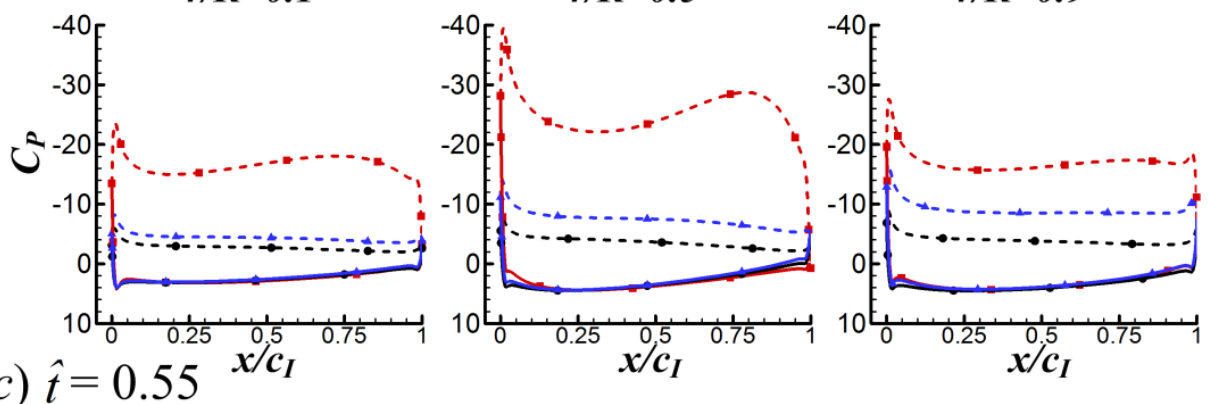

(c) $\hat{t}=0.55$

$r / R=0.1$

$r / R=0.5$

$r / R=0.9$
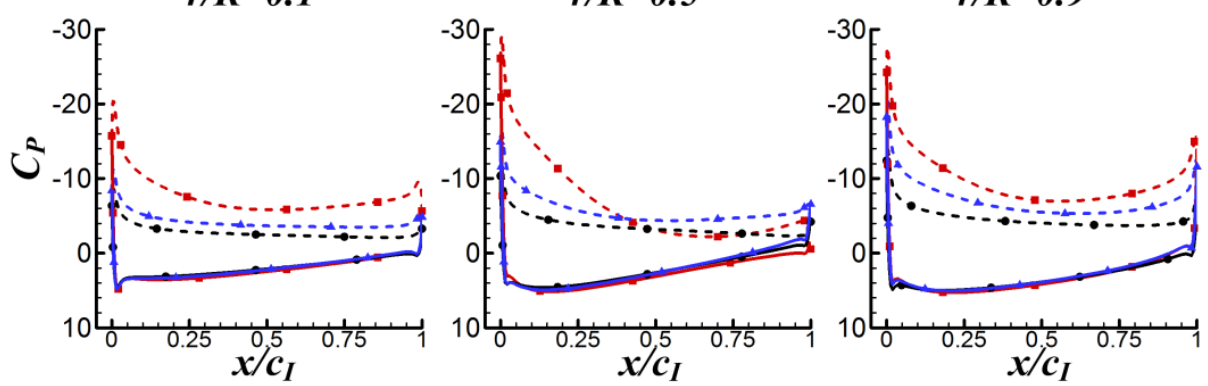

Figure 12. Surface pressure distributions of the three cases at various spanwise distribution on the lower surface and the upper surface, respectively). 
Full

(a) $\hat{t}=0.52$

(d) $\hat{t}=0.52$

$\stackrel{U}{\rightarrow}$

Partial

(g) $\hat{t}=0.52$
Single

$\stackrel{U}{\rightarrow}, \stackrel{U}{\rightarrow}$

(h) $\hat{t}=0.53$

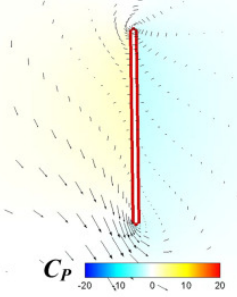

$\stackrel{U}{\rightarrow}$

$\underline{U}$

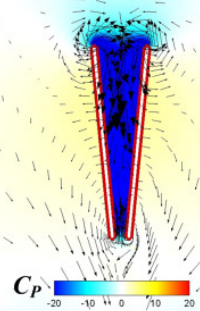

(c) $\hat{t}=0.55$

$\underset{U}{\longrightarrow}$

(f) $\hat{t}=0.55$

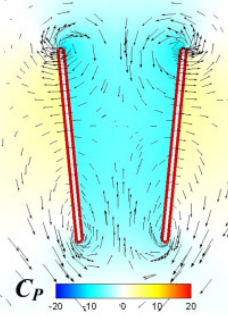

$\stackrel{U}{\longrightarrow}$
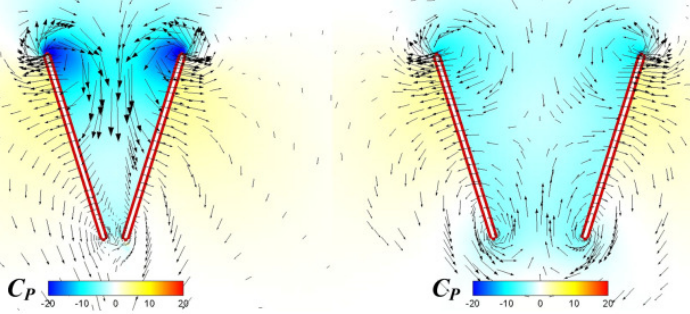

(i) $\hat{t}=0.55$

$\stackrel{U}{\rightarrow}$

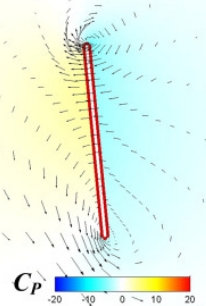

,

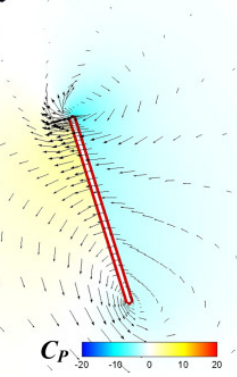

Figure 13. Velocity vectors and pressure distributions of the FCF case (a-c), 

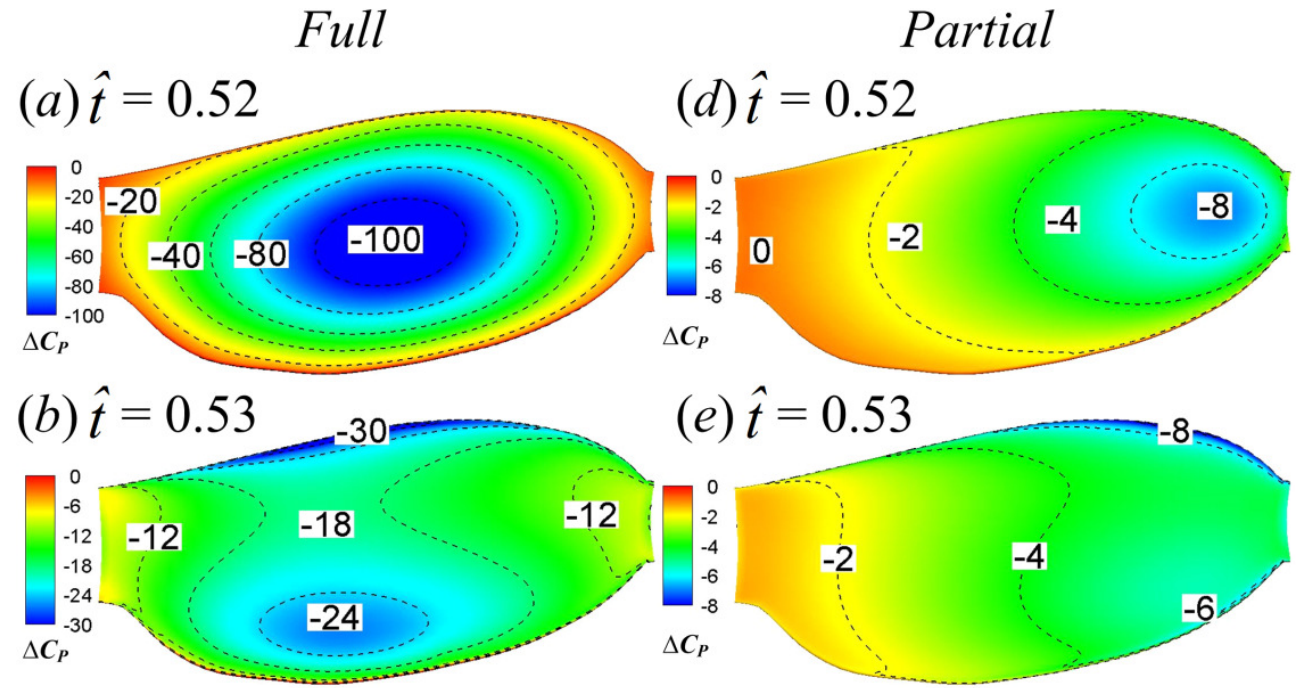

(c) $\hat{t}=0.55$

489

490

491

492

493

494

495

496

497

498

499

500

501

502

\section{(4) Interference effects between wings in the subsequent translation after fling}

Though the lift-enhancing effect of wing-wing interaction is mostly restricted to the clap-fling phase, it is still visible in the translational phase after fling (see Fig. 6b). Compared with the SW case, the average $C_{L}$ during $\hat{t}=0.61-1.0$ of the FCF case and the PCF case is increased by $20 \%$ and $10 \%$ respectively. Because lift is increased in the FCF and PCF cases for similar reasons and the lift-enhancing effect is more obvious in the FCF case, we only compare the FCF and SW cases. Since the attached LEV is the dominant lift-generating mechanism, the LEV strength may vary between the two cases. Figure 15 shows the iso-vorticity surface plots (top view) and their corresponding spanwise vorticities

Peer) reviewing PDF | (2016:09:13484:1:1:CHECK 22 Dec 201369) 
503 at the mid span location at $\hat{t}=0.62,0.64$ and 0.66 of the FCF case (a-c) and the

504 SW case (d-f). After the start of fling, a strong $\mathrm{LEV}_{1}$ is generated and then it is

505 shed from the wing in the later part of fling due to the angular deceleration of wing.

506 As the wing continues to rotate, the shed $\mathrm{LEV}_{1}$ peels away from the upper surface

507 of wing and a new $\mathrm{LEV}_{2}$, begins to form and grows quickly (see Fig. 15).

508 Comparing the LEV strength in the FCF and SW cases shows that the strength of

509 both $\mathrm{LEV}_{1}$ and $\mathrm{LEV}_{2}$ in the FCF case is stronger. A possible explanation for this is

510 as follows. A strong low pressure region between wings is created in the

511 two-winged case which sucks more fluid into the opening gap around leading edge

512 and thus creates stronger LEV than that in the single-winged case. Collectively,

513 the lift enhancement after fling in the two-winged cases is attributed partially to

514 the subsequent effect of old LEV generated in the fling phase and partially to the

515 new LEV generated in the translation phase. 
Full

Single

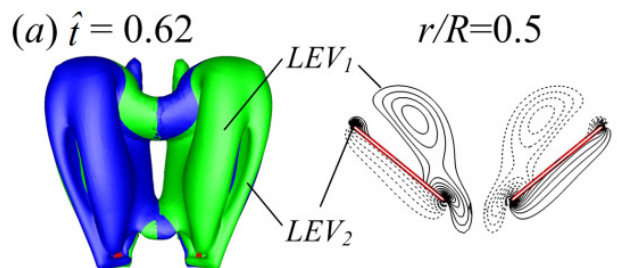

(d) $\hat{t}=0.62$

$r / R=0.5$

(b) $\hat{t}=0.64$

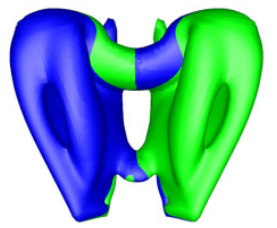

(c) $\hat{t}=0.66$

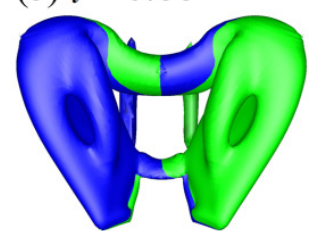

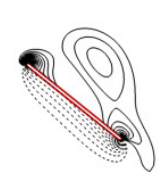

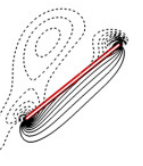

(e) $\hat{t}=0.64$

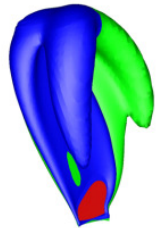

(f) $\hat{t}=0.66$
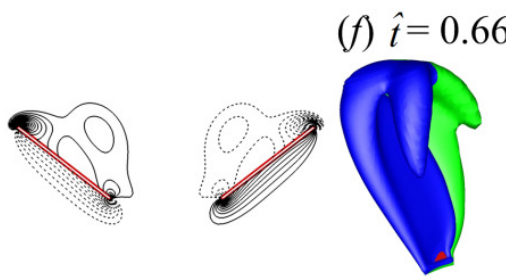
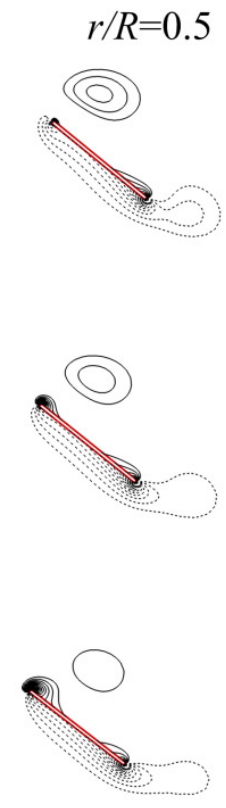

Figure 15. Iso-vorticity surface plots (top view; the magnitude of the non-dimensional vorticity is 3) and their corresponding spanwise vorticities (the magnitude of the vorticity at the outer contour is 2 and the contour interval is 1) at half-wing length for $\hat{t}=0.62,0.64$ and 0.66 of the FCF case (a-c) and SW case (d-f). Vortex structures are shaded by spanwise vorticity to indicate direction: green is negative and blue is positive.

Note that in this study, $\alpha$ is assumed to be constant in the translation phase and varies only at stroke reversal. How will the obtained results vary if a different $\alpha$ variation is employed? To see this, we have done some additional computations on a sinusoidal variation of $\alpha$ (see Supplementary S2). The results show the following. The interference effect between wings is no longer restricted to the dorsal stroke reversal but extends to the translation phase before and after dorsal stroke reversal, possibly 


\section{Manuscript to be reviewed}

531 due to the different $\alpha$ variation. Moreover, since the wing root distance is increased,

532 the force enhancement becomes similar among the two-wing cases: $\bar{C}_{L}$ and $\bar{C}_{D}$

533 are increased by about $13 \%$ and $8 \%$ respectively, compared to the one-winged case.

534 In the above discussions, only one PCF case with certain root distance and

535 angular excursion is investigated. Since the PCF case, in terms of power efficiency,

536 performs better than the FCF and SW cases, is there a best partial clap-fling

537 motion? To see this, we have done some additional computations in which other

538 root distance and angular excursion combinations are considered (see

539 Supplementary S3). A new variable, the angular distance $\theta$ between the spanwise

540 axis of each wing and the mid plane at $\hat{t}=0.5$ (the end of the clap phase), is

541 introduced to characterize different motions (Fig. S4a). In all the clap-fling

542 motions, the distance between wing tips at $\hat{t}=0.5$ is kept constant at $d=0.1 c$. By

543 this definition, the FCF and PCF cases studied correspond to the $\theta=0^{\circ}$ case and

$544 \theta=7.9^{\circ}$ case respectively. The other four partial clap-fling motions correspond to

$545 \theta=2^{\circ}, \theta=4^{\circ}, \theta=6^{\circ}$ and $\theta=10^{\circ}$, respectively. The results and detailed analysis are

546 given in Supplementary S3. The main results are as following. The lift

547 enhancement is largest in the case of $\theta=0^{\circ}$ (the FCF case), but the drag is also the

548 largest (i.e. the energy consumption is much larger than that of the SW case).

549 When $\theta$ is increased to $\theta=2^{\circ}$, only a slight increase, one can have a large $\bar{C}_{L}$

550 augmentation without a large $\bar{C}_{D}\left(\bar{C}_{L}\right.$ is increased by $21 \%$ and $\bar{C}_{L} / \bar{C}_{D}$ is just

551 slightly lower than that of the SW case). When $\theta$ is further increased, $\bar{C}_{L}$ and

$552 \bar{C}_{D}$ continue to decrease gradually while $\bar{C}_{L} / \bar{C}_{D}$ stays almost constant. This 


\section{Manuscript to be reviewed}

553 suggests that the two wings should be close enough, but not too close, to have a 554 good interference effect.

555

\section{Conclusion}

557 (1) During the clap phase, the wings clap together and create a high pressure region 558 in the closing gap between wings, greatly increasing the positive pressure on the 559 lower surface of wing, while pressure on the upper surface is almost unchanged by 560 the interaction; during the fling phase, the wings fling apart and create a low pressure 561 region in the opening gap between wings, greatly increasing the suction pressure on 562 the upper surface of wing, while pressure on the lower surface is almost unchanged 563 by the interaction.

564 (2) In the full clap-fling case, the interference effect between wings is most drastic at 565 the end of clap phase and the start of the fling phase: two sharp force peaks (8-9 566 times larger than that of the single-winged case) are generated. However, as the wing 567 section is nearly vertical, the normal force peaks are mostly manifested as drag and 568 barely as lift of the wing.

569 (3) In the partial clap-fling case, only the outer parts of wings are in close proximity 570 and the wing separation increases from wing tip to wing root. So the wing-wing 571 interaction effect in the partial clap-fling case is much weaker than that in the full 572 clap-fling case, avoiding the generation of huge drag. The partial clap-fling is a more 573 practical choice for tiny insects to employ: it can augment the mean lift coefficient by about $12 \%$ without suffering any efficiency degradation when compared to the 


\section{Manuscript to be reviewed}

575 single-winged case.

576

577 References

578 1. Dudley, R. Section 7.1.1 in The Biomechanics of Insect Flight: Form, Function, $579 \quad$ Evolution. Princeton University Press (2002).

$580 \quad$ 2. Walker, S. M., Thomas, A. L. . \& Taylor, G. K. Photogrammetric reconstruction of high-resolution surface topographies and deformable wing kinematics of tethered locusts and free-flying hoverflies. J. R. Soc. Interface 6, $351-366$ (2009).

3. Cheng, B., Deng, X. \& Hedrick, T. L. The mechanics and control of pitching manoeuvres in a freely flying hawkmoth (Manduca sexta). J. Exp. Biol. 214, 4092-106 (2011).

4. Sun, M. \& Lan, S. L. A computational study of the aerodynamic forces and power requirements of dragonfly (Aeschna juncea) hovering. J. Exp. Biol. 207,

5. Dudley, R. \& Ellington, C. P. Mechanics of Forward Flight in Bumblebees: I. Kinematics and Morphology. J. Exp. Biol. 148, 19-52 (1990).

6. Liu, Y. P. \& Sun, M. Wing kinematics measurement and aerodynamics of hovering droneflies. J. Exp. Biol. 211, 2014-2025 (2008). 


\section{Manuscript to be reviewed}

597

598

599

600

601

602

603

604

605

606

607

608

609

610

611

612

613

614

615

616

617

618

fruitflies. Phys. Fluids 27, 0-22 (2015).

9. Lua, K. B., Lee, Y. J., Lim, T. T. \& Yeo, K. S. Aerodynamic Effects of Elevating Motion on Hovering Rigid Hawkmothlike Wings. AIAA J. 54, 22472264 (2016).

10. Lu, H., Lua, K. B., Lee, Y. J., Lim, T. T. \& Yeo, K. S. Ground effect on the aerodynamics of three-dimensional hovering wings. Bioinspir. Biomim. 11, 66003 (2016).

11. Terry, I. Thrips and weevils as dual, specialist pollinators of the Australian cycad Macrozamia communis (Zamiaceae). Int. J. Plant Sci. 162, 1293-1305 (2001).

12. Crespi, B. J., Carmean, D. a \& Chapman, T. W. Ecology and evolution of galling thrips and their allies. Annu. Rev. Entomol. 42, 51-71 (1997).

13. Austin, A. D. \& Dowton, M. Hymenoptera: Evolution, Biodiversity and Biological Control. (CSIRO, 2000).

14. Weis-Fogh, T. Quick Estimates of Flight Fitness in Hovering Animals, Including Novel Mechanisms for Lift Production. J. Exp. Biol. (1973).

15. Santhanakrishnan, A., Robinson, A. K, Jones, S., Low, A. A., Gadi, S., Hedrick, T. L. \& Miller, L. A. Clap and fling mechanism with interacting porous wings in tiny insect flight. J. Exp. Biol. 217, 3898-3909 (2014).

16. Cheng, X. \& Sun, M. Wing-kinematics measurement and aerodynamics in a small insect in hovering flight. Sci. Rep. 6, 25706 (2016).

17. Wang, Z. J. Two dimensional mechanism for insect hovering. Phys. Rev. Lett. 


\section{Manuscript to be reviewed}

619

620

621

622

623

624

625

626

627

628

629

630

631

632

633

634

635

636

637

638

639

640

85, 2216-2219 (2000).

18. Miller, L. A. \& Peskin, C. S. When vortices stick: An aerodynamic transition in tiny insect flight. J. Exp. Biol. 207, 3073-3088 (2004).

19. Sun, J. H. W. and M. Unsteady aerodynamic forces of a flapping wing. J. Exp. Biol. 207, 1137-1150 (2004).

20. Weis-Fogh, T. Unusual Mechanisms for the Generation of Lift in Flying Animals. Sci. Am. 233, 80-87 (1975).

21. Ellington, C. P. The Aerodynamics of Hovering Insect Flight. III. Kinematics. Philos. Trans. R. Soc. B Biol. Sci. 305, 41-78 (1984).

22. Miller, L. A. \& Peskin, C. S. Flexible clap and fling in tiny insect flight. J. Exp. Biol. 212, 3076-3090 (2009).

23. Murphy, D. W., Adhikari, D., Webster, D. R. \& Yen, J. Underwater flight by the planktonic sea butterfly. J. Exp. Biol. 219, 535-543 (2016).

24. Sunada, S. \& Kawachi, K. Performance of a Butterfly in Take-Off Flight. $J$. Exp. Biol. 277, 249-277 (1993).

25. Cooter, R. J. \& Baker, P. S. Weis-Fogh clap and fling mechanism in Locusta. Nature 269, 53-54 (1977).

26. Lighthill, M. J. On the Weis-Fogh mechanism of lift generation. J. Fluid Mech. 60, 1 (1973).

27. Edwards, R. H. \& Cheng, H. K. The separation vortex in the Weis-Fogh circulation-generation mechanism. J. Fluid Mech. 120, 463 (1982).

28. Ellington, C. P. The Aerodynamics of Hovering Insect Flight. IV. 


\section{Manuscript to be reviewed}

641

642

643

644

645

646

647

648

649

650

651

652

653

654

655

656

657

658

659

660

661

662

Aeorodynamic Mechanisms. Philos. Trans. R. Soc. B Biol. Sci. 305, 79-113

(1984).

29. Wu, J. C. \& Chen, H. Unsteady aerodynamics of articulate lifting bodies. in 2nd Applied Aerodynamics Conference (American Institute of Aeronautics and Astronautics, 1984).

30. Kolomenskiy, D., Moffatt, H. K., FARGE, M. \& Schneider, K. The LighthillWeis-Fogh clap-fling-sweep mechanism revisited. J. Fluid Mech. 676, 572606 (2011).

31. Maxworthy, T. Experiments on the Weis-Fogh mechanism of lift generation by insects in hovering flight. Part 1. Dynamics of the 'fling'. J. Fluid Mech. 93, 47 (1979).

32. Spedding, G. R. \& Maxworthy, T. The generation of circulation and lift in a rigid two-dimensional fling. J. Fluid Mech. 165, 247 (1986).

33. Lehmann, F. O. \& Pick, S. The aerodynamic benefit of wing-wing interaction depends on stroke trajectory in flapping insect wings. J. Exp. Biol. 210, 13621377 (2007).

34. Ro, K. \& Tsutahara, M. Numerical analysis of unsteady flow in the Weis-Fogh mechanism by the 3D discrete vortex method with GRAPE3A. J. Fluids Eng. Asme 119, 96-102 (1997).

35. Sun, M. \& Yu, X. Flows around two airfoils performing fling and subsequent translation and translation and subsequent clap. Acta Mech. Sin. 19, 103-117 (2003). 


\section{Manuscript to be reviewed}

663 36. Miller, L. A. \& Peskin, C. S. A computational fluid dynamics of 'clap and fling'

664

665

666

667

668

669

670

671

672

673

674

675

676

677

678

679

680

681

682

683

684 in the smallest insects. J. Exp. Biol. 208, 195-212 (2005).

37. Sun, M. \& Yu, X. Aerodynamic Force Generation in Hovering Flight in a Tiny Insect. AIAA J. 44, 1532-1540 (2006).

38. Kolomenskiy, D., Moffatt, H. K., Farge, M. \& Schneider, K. Vorticity generation during the clap-fling-sweep of some hovering insects. Theor. Comput. Fluid Dyn. 24, 209-215 (2010).

39. Kolomenskiy, D., Moffatt, H. K., Farge, M. \& Schneider, K. Two- and three-dimensional numerical simulations of the clap-fling-sweep of hovering insects. J. Fluids Struct. 27, 784-791 (2011).

40. Arora, N., Gupta, A., Sanghi, S., Aono, H. \& Shyy, W. Lift-drag and flow structures associated with the 'clap and fling' motion. Phys. Fluids 26, (2014).

41. Jones, S. K., Laurenza, R., Hedrick, T. L., Griffith, B. E. \& Miller, L. A. Lift vs. drag based mechanisms for vertical force production in the smallest flying insects. J. Theor. Biol. 384, 105-120 (2015).

42. Lehmann, F.O. Sane, S. P. \& Dickinson, M. The aerodynamic effects of wing-wing interaction in flapping insect wings. J. Exp. Biol. 208, 3075-3092 (2005).

43. Lua, K. B., Lai, K. C., Lim, T. T. \& Yeo, K. S. On the aerodynamic characteristics of hovering rigid and flexible hawkmoth-like wings. Exp. Fluids 49, 1263-1291 (2010).

44. Lua, K. B., Lim, T. T. \& Yeo, K. S. Scaling of Aerodynamic Forces of 
685

686

687

688

689

690

Three-Dimensional Flapping Wings. AIAA J. 52, 1095-1101 (2014).

45. Ellington, C. P. Non-Steady-State Aerodynamics of the Flight of Encarsia formosa in Swimming and Flying in Nature. Vol. 2 (edited by Wu, T. Y., Brokaw, C. J. \& Brenen, C.) 783-796 (Springer, 1975).

46. Sun, M. \& Tang, J. Unsteady aerodynamic force generation by a model fruit fly wing in flapping motion. J. Exp. Biol. 205, 55-70 (2002). 